\title{
In vivo RNAi screen identifies NLK as a negative regulator of mesenchymal activity in glioblastoma
}

\author{
Jason K. Sa ${ }^{1,2,3, *}$, Yeup Yoon ${ }^{1, *}$, Misuk Kim ${ }^{2,3, *}$, Yeonghwan $\mathrm{Kim}^{4, *}$, Hee Jin \\ Cho $^{1,2,3}$, Jin-Ku Lee ${ }^{2,3}$, Gi-Soo Kim ${ }^{3}$, Suji Han ${ }^{1,2,3}$, Woon Jin Kim ${ }^{1,2,3}$, Yong Jae \\ Shinn $^{2,3}$, Kyeung Min Joo ${ }^{1,2,3}$, Patrick J. Paddison' ${ }^{5}$ Tohru Ishitani ${ }^{6}$, Jeongwu Lee ${ }^{4}$, \\ Do-Hyun Nam $^{1,2,3,7}$ \\ ${ }^{1}$ Graduate School of Health Science \& Technology, Samsung Advanced Institute for Health Science \& Technology (SAIHST), \\ Sungkyunkwan University, Seoul, Korea \\ ${ }^{2}$ Samsung Biomedical Research Institute, Samsung Medical Center, Seoul, Korea \\ ${ }^{3}$ Institute for Refractory Cancer Research, Samsung Medical Center, Seoul, Korea \\ ${ }^{4}$ Department of Stem Cell Biology and Regenerative Medicine, Lerner Research Institute, Cleveland Clinic, Cleveland, OH, USA \\ ${ }^{5}$ Human Biology Division, Fred Hutchinson Cancer Research Center, Seattle, WA, USA \\ ${ }^{6}$ Division of Cell Regulation Systems, Medical Institute of Bioregulation, Kyushu University, Fukuoka, Japan \\ ${ }^{7}$ Department of Neurosurgery, Samsung Medical Center, Sungkyunkwan University School of Medicine, Seoul, Korea \\ *These authors have contributed equally to this work
}

Correspondence to:

Do-Hyun Nam, e-mail: nsnam@skku.edu

Jeongwu Lee, e-mail: leej7@ccf.org

Keywords: glioblastoma, RNA interference screen, Nemo-like kinase, stemness, mesenchymal

Received: April 23, $2015 \quad$ Accepted: May 06, $2015 \quad$ Published: May 19, 2015

\section{ABSTRACT}

Glioblastoma (GBM) is the most lethal brain cancer with profound genomic alterations. While the bona fide tumor suppressor genes such as PTEN, NF1, and TP53 have high frequency of inactivating mutations, there may be the genes with GBM-suppressive roles for which genomic mutation is not a primary cause for inactivation. To identify such genes, we employed in vivo RNAi screening approach using the patient-derived GBM xenograft models. We found that Nemo-Like Kinase (NLK) negatively regulates mesenchymal activities, a characteristic of aggressive GBM, in part via inhibition of WNT/ $\beta$-catenin signaling. Consistent with this, we found that NLK expression is especially low in a subset of GBMs that harbors high WNT/mesenchymal activities. Restoration of NLK inhibited WNT and mesenchymal activities, decreased clonogenic growth and survival, and impeded tumor growth in vivo. These data unravel a tumor suppressive role of NLK and support the feasibility of combining oncogenomics with in vivo RNAi screen.

\section{INTRODUCTION}

Glioblastoma (GBM) is the most common and lethal primary brain tumor [1]. Unfortunately, the current standard-of-care for GBM patients provides only palliation with a median survival of 14.6 months in spite of surgery, chemotherapy, and radiation [2,3]. Molecular circuits and factors behind its aggressive behaviors and malignant characteristics remain incompletely understood. One of the key components for GBM malignancy is the loss of functional tumor suppressor genes. Tumor suppressors act in signaling networks that restrict cellular proliferation and present barriers to malignant transformation. Even though tumor suppressor genes per se may not be considered optimal as drug targets, their loss of function could create cellular dependency which could be exploited therapeutically [4].

Tumor suppressors are disabled by combinations of point mutations, genomic deletions and promoter methylation. Indeed, recent large-scale genomic studies on patient-derived GBM specimens conclusively showed various genomic copy number alterations and mutations $[5,6]$. For example, genomic deletions and mutations of TP53, NF1, and PTEN are frequently found 
in various cancers including GBMs. Because mutations in one allele are often followed by deletion of the other, somatic deletions in human cancers often pinpoint tumor suppressor genes that function as 'drivers' of tumor evolution. However, the large-scale genomic analyses also revealed the large list of genes that may have "tumorsuppressive" roles but the frequencies of inactivating mutations are relatively uncommon [6]. We set out to determine the functional roles of these candidate genes in gliomagenesis.

By combined in silico analyses of genomic copy variation $(\mathrm{CNV})$ and transcriptome profiling of human GBM specimens, we have derived the gene sets whose genome copy numbers and expression levels are significantly low in GBM specimens. To interrogate the functional roles of the candidate genes in a relevant and systemic manner, we have adapted stable RNA interference (RNAi) screening technology in vivo. By implementing loss-of-function genetics in the setting of in vivo GBM models that mimic the biology of human GBM, we validated the effects of our candidate genes on tumor growth. Here, we have identified NLK as a putative tumor suppressor gene and demonstrated that NLK plays a critical role in tumor restriction through regulation of Wnt/ beta-catenin pathway and mesenchymal activity in GBM.

\section{RESULTS}

\section{In vivo RNAi screen utilizing human GBM-derived xenograft models}

To identify putative tumor suppressor genes in GBM, we first generated the candidate gene sets by utilizing genomic and transcriptome data of patientderived GBM specimens $(n=228)$ publicly available from Rembrandt. We selected candidate genes by the levels of genomic deletions and low mRNA expressions in tumors compared to non-tumor brains $(n=28)$. The cut-off for genomic deletion was less than 1.6 of genomic copy number (compared to 2.0 in normal cells) in more than $15 \%$ of the GBM specimens. Differential expression of a given gene in GBM and non-tumor brains was determined by Affymetrix array data and statistically validated. Through TCGA and cBioPortal database, we determined the reported frequency of somatic mutations (Figure S1). As expected, these candidate gene sets include well-known tumor suppressor genes, PTEN and $C D K N 2 A / B$ (Table S1). Loss of heterozygosity (LOH) on chromosome 10 is known to be the most frequent genetic alteration in GBMs and it has been suggested that multiple tumor suppressor genes may exist on chromosome 10 [7]. Consistent with this, majority of the candidate gene sets were located on chromosome 10 . We have generated a shRNA pool directed against these gene sets by selecting individual shRNA lentiviral clones from Cold Spring
Harbor shRNA libraries. On average, there were 5 to 7 shRNA clones for each targeted genes.

Our experimental scheme for in vivo RNAi screen was summarized in Figre 1A. We aimed to achieve that each tumor cell would be integrated with a single unique shRNA clone. Once these cells were injected for orthotopic tumor generation in mouse brains, a subset of tumor cells would outgrow presumably due to the selective growth advantages conferred by a specific shRNA (Figure 1A). As each shRNA vector was uniquely labeled with a DNA barcode, sequencing analysis of the resultant tumors will inform the relative contribution of each clone in tumor. By using GFP expressing shRNA vectors, we determined the optimal multiplicity of infection (MOI) to ensure that most cells would intake a single copy of the lentiviral shRNA (data not shown).

We have previously established a series of patientderived GBM xenografts and demonstrated that these tumors maintain the genomic and biological characteristics of the parental GBM tumors [8]. In these GBM models, intracranial injection of 100, 000 tumor cells was sufficient to generate tumors with near $100 \%$ tumor take efficacy. Complexity of shRNA pools and the number of different shRNAs in a given population are crucial factors of in vivo RNAi screen [9]. Through titration and cell tracing analyses, we determined that an average representation of approximately 500 cells per shRNA construct could provide a reliable index to ensure the detection of each shRNA clone and prevent any initial depletion of certain shRNAs prior to intracranial injection procedure and the overall screening process (data not shown). With these optimization processes, we have constructed the lentiviral shRNA pool containing approximately 200 individual shRNAs, transduced into patient-derived GBM cells, and injected the resulting cells into recipient mice or collected immediately as the control population. Three different patient-derived GBM cells (GBM 1 to 3, 5 mice for each GBM) were used in this screen to cover the diverse GBM phenotypes. By using ArrayCGH and Whole Exome Sequencing, we characterized genomic profiles of these tumors. All three GBMs contain homozygous genomic deletion of $C D K N 2 A$. GBM 1 and 2 harbor wild-type PTEN gene, while GBM 3 does not express any PTEN proteins. Once body weight loss and changes in health status including neurological signs have been observed, xenograft tumors were harvested for the extraction of tumor DNA. After PCR amplification of shRNA hairpins from tumor-derived genomic DNA, hairpin representations were analyzed using high-throughput sequencing technology.

We have acquired a list of shRNA hairpins that are enriched for more than 3-fold in xenograft tumor cells compared to the initial control populations (Figure 1B). To avoid false positive results derived by random accumulation of specific shRNAs and/or potential off-target effects, we set additional criteria for the enriched candidate 


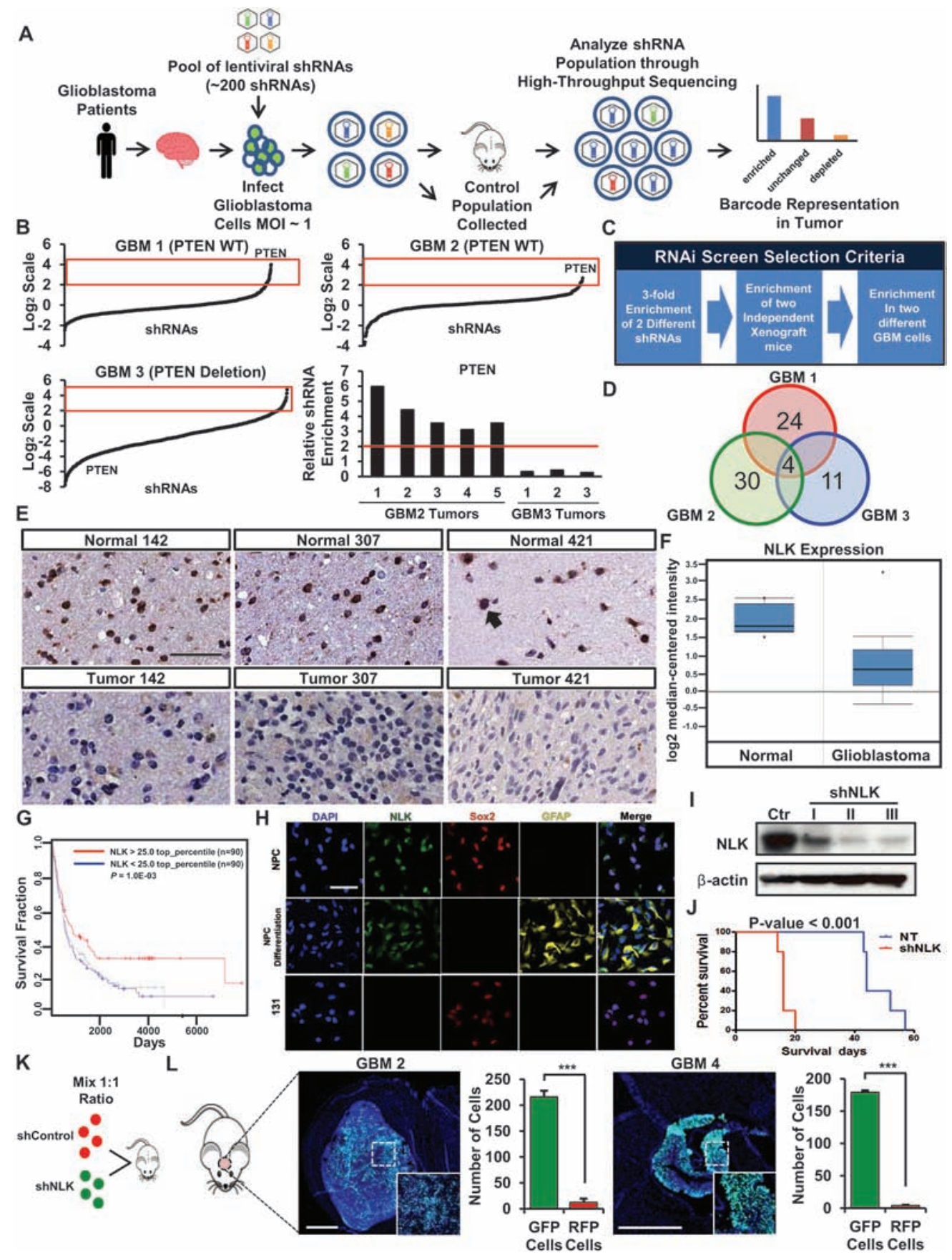

Figure 1: In vivo RNA interference screening identifies putative tumor suppressors in GBM. A. Schematic representation of in vivo RNAi screening. Patient-derived GBM cells are transduced with the shRNA library pool and injected into the mice brains. Tumors were harvested and shRNAs were PCR amplified and deep sequenced to identify candidate "hits". B. Analysis of the shRNAs recovered from our RNAi screening. Data are normalized to the Control population and plotted in Log2 scale. Red box indicates enriched shRNAs. Enrichment of PTEN shRNAs in GBM 2 tumors (PTEN WT) compared to the GBM 3 tumors (PTEN deletion). C. A selection criteria for candidate hits from the RNA interference screen. D. Candidate "hits" from the screen. E. Immunohistochemistry of NLK on TissueMicroArray (TMA) containing $88 \mathrm{GBM}$ samples and 32 normal brain samples. Scale bar, $100 \mu \mathrm{m}$. F. Oncomine microarray data analysis for NLK expression in glioblastoma versus normal brain tissues. $(p<0.001, n=525)$ G. Analysis of REMBRANDT public dataset on glioma patient survival in accordance with NLK ${ }^{\text {high }}$ and NLK $^{\text {low }}$ expressions $(n=90$ each). H. Representative confocal microscopy images of immunofluorescence (IF) staining of NLK, Sox2, and GFAP in normal neural progenitor cells (NPC), differentiated NPC, and GBM cells. Scale bar, $100 \mu \mathrm{m}$. I. Immunoblots of NLK in patient-derived GBM cells transduced with Control or shRNA NLK. J. KaplanMeier survival curves of Control vs shRNA NLK. K-L, Schematic representation of dual-color competition assay in vivo. K A total of 50,000 cells from a 1:1 mixture of RFP-labeled shControl cells (red) and GFP-labeled shNLK cells (green) were implanted into mouse brains. L Immunofluorescence images of cryo-sectioned mouse brains. Scale bar, $500 \mu \mathrm{m}$. Bar graph represents the number of GFP and RFP positive cells that were counted in different spots of the tumor that were selected randomly. $(+\mathrm{SD}, n=4)$. 
genes; 1) at least 2 different shRNAs should be detected for a single gene, 2) the enriched shRNAs should be observed in at least two independent xenograft tumors, and 3 ) the enriched shRNAs should be observed in at least two different GBM cells (Figure 1C). PTEN-shRNA harboring cells were enriched for more than 5 fold in GBM1 and GBM2 but not in GBM3 (Figure 1B). As predicted considering homozygous genomic deletion of $C D K N 2 A$ in these tumors, $C D K N 2 A$ shRNAs were not enriched in any screens. Collectively, these results support the fidelity of our in vivo RNAi screen and validation procedures.

In addition to PTEN, in vivo RNAi screens further identified 4 candidate genes, including MXI1, NLK, and MAPK8 (Figure 1D). As previous studies have shown that MXI1 and PTEN were both involved in GBM as tumor suppressor genes for their abilities to regulate tumor cell growth, migration, and apoptosis [10-13], we decided to focus on NLK. Anti-tumorigenic effects of NLK have been characterized in different cancer types [14-16], but relatively little is understood about the role of NLK in GBM. While NLK has been implicated in Wnt signaling pathway, a critical regulator in GBM [17], it remains unclear how NLK-WNT signaling axis affects stem cell associated phenotypes or whether NLK loss is specifically linked to GBM subtypes.

First, we determined the expression levels of NLK protein in matched GBM and adjacent non-tumor brain specimens by tissue microarray (TMA). In contrast to high NLK expression in non-tumor tissues, GBM tissues have little or no expression (Figure 1E). Quantitation of immunohistochemical analyses using the sections derived from 88 GBM specimens and non-tumor brain tissues revealed significant difference in NLK expression. To further validate these findings, we surveyed the expression levels of NLK mRNA in glioma specimens utilizing Oncomine and Rembrandt databases [18, 19] and found that NLK mRNA levels are significantly lower in GBMs compared to non-tumor brain tissues (Figure 1F). When we stratified glioma patients by NLK mRNA levels, a subset of glioma patients with top $25 \%$ of NLK mRNA survived significantly longer than the remaining patients, suggesting a potential association between NLK levels and patient survival (Figure 1G).

As NLK is expressed in normal brain tissues, we further determined expression of NLK in normal neural progenitor cells (NPCs) and their differentiated progenies. Regardless of their differentiated status, SOX2- positive NPCs and astrocyte marker GFAP-positive cells both expressed NLK. In contrast, the patient-derived GBM cells (131) displayed little or no NLK expression (Figure 1H).

Some GBM specimens including the samples that were used for in vivo RNAi screen express NLK, albeit much lower compared to normal brain tissues, as shown in Figure 1F. To validate RNAi screening results, we further inhibited NLK by shRNA-mediated knockdown and evaluated its effects on tumor growth (Figure 1I-1L).
Control or NLK shRNA expressing tumor cells were implanted orthotopically and animal survival was determined. Mice injected with NLK shRNA expressing cells died much faster than the mice with control cells (Figure 1J). Additionally, we performed in vivo growth competition assays in which control (RFP labeled) or NLK shRNA tumor cells (GFP labeled) were mixed and co-injected into mice brains. We harvested the resulting tumors and performed FACs analysis and histological analysis. Although the same number of tumor cells was injected, more than $95 \%$ of the resulting tumor cells were derived from GFP-positive, NLK shRNA expressing cells (Figure 1L). Together, these results support that NLK inhibition facilitates in vivo tumor growth.

\section{NLK impedes clonogenic growth and stem-like properties of GBM}

To elucidate the function of NLK in GBM, we first investigated its ability to regulate cell proliferation in GBM by ectopic expression of NLK. Three patientderived GBM cells 387, 827, and 131 express low levels of NLK proteins. Transduction with lentivirus expressing NLK-wild type (WT) significantly increased the expression of NLK in these cells (Figure 2A). Shortterm proliferation kinetics of tumor cells, determined by MTT assays, demonstrated a significant decrease of cell proliferation in NLK-transduced GBM cells compared to the control groups (Figure 2B).

Cancer stem/initiating cells (CSCs) are functionally defined by their enhanced ability to initiate tumors in vivo. Although some cancers may not follow the CSC model, numerous studies support that GBMs harbor a subpopulation of highly tumorigenic, stem-like cells (GSCs) [20-23]. GSC population is enriched with selfrenewal capacity, which may contribute to the aggressive behavior of GBM [24-26]. Given an anti-proliferative effect of NLK, we reasoned that NLK could influence stem-like properties of GBM. Clonogenic growth of GBM cells as spheroids is an indicator of stem-like GBM cells in vitro. We plated tumor cells at the clonal density ranging from 1 to 50 cells per well and monitored clonogenic growth of tumor cells with or without overexpression of NLK. The estimated frequencies of clonogenic cells were significantly high in the control tumor cells $(1 / 4$, $1 / 6$, and $1 / 9$ for 131,387 , and 827 cells, respectively) than NLK-overexpressing cells $(1 / 95,1 / 27$, and 1/35 for 131,387 , and 827 cells, respectively), implicating the role of NLK in clonogenic growth of GBM (Figure 2C and 2D). To further corroborate the association between NLK and stemness, we determined the expression levels of well-known GSC-associated factors such as Nanog and Sox2. Results from real-time RT-PCR, immunoblot, and immunofluorescence analysis revealed significantly low levels of Nanog and Sox 2 in NLK-overexpressing cells compared to the control cells (Figure 2E-2G). 


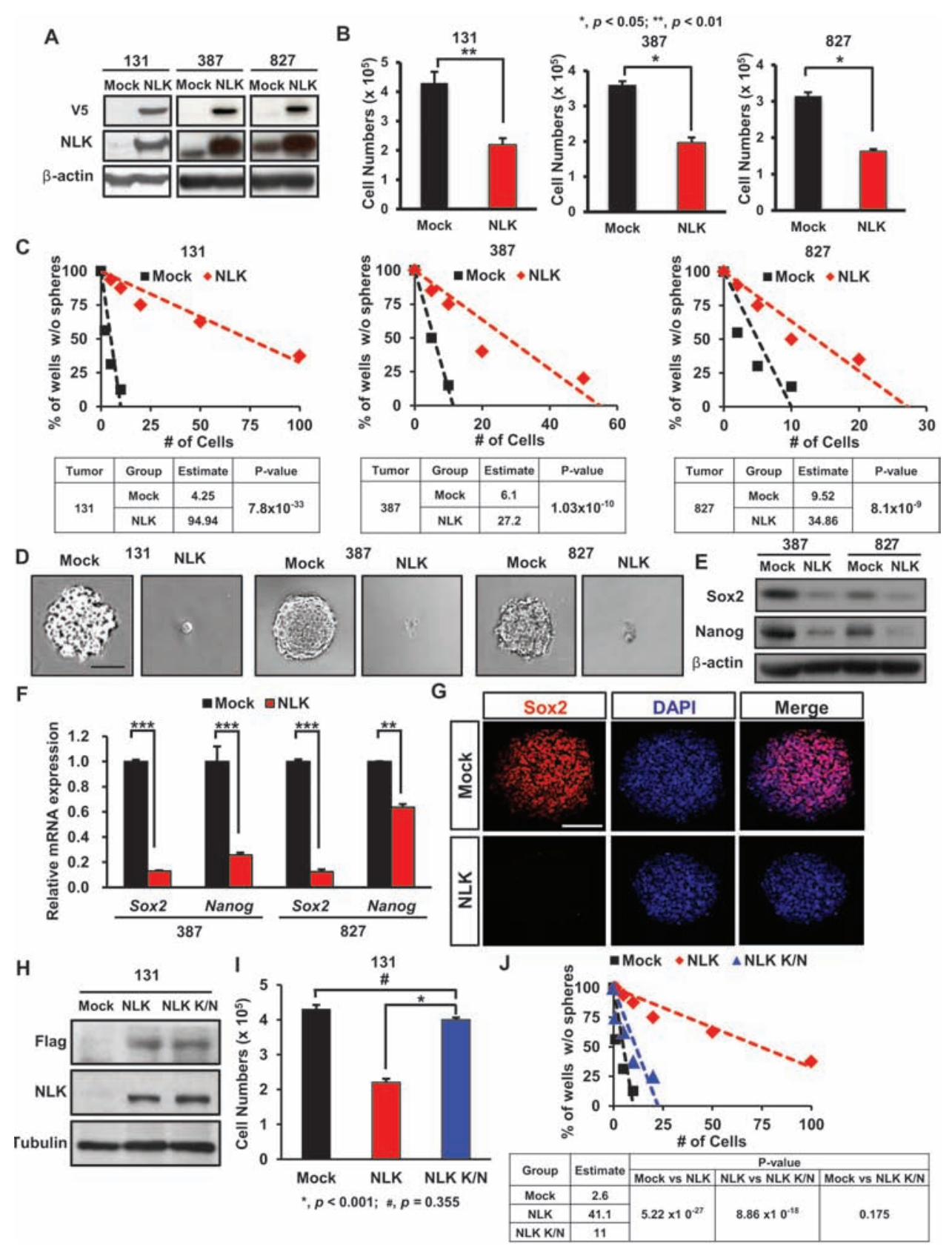

Figure 2: NLK regulates proliferation and stem cell-like properties in patient-derived primary GBMs. A. Immunoblots of V5-NLK and NLK in patient-derived GBM cells transduced with Mock control or NLK-WT vector. B. Comparison on the effects of NLK on in vitro proliferation. ( $+\mathrm{SD}, n=5$ ) $\mathbf{C}$. Limiting dilution assays (LDA) for in vitro tumor sphere formation. LDA clonogenic significance is measured by the linear regression analysis. D. Overexpression of NLK suppresses tumor sphere formation. Scale bar, $100 \mu \mathrm{m}$. E. Immunoblots of Sox 2 and Nanog in NLK-WT or mock transduced GBM cells. F. Real-time RT-PCR analysis to determine the effects of NLK on mRNA expression levels of Soxz and Nanog. G. Representative confocal images of immunofluorescence (IF) staining of Sox2 in spheroids. Scale bar, $100 \mu \mathrm{m}$. H. Immunoblots of anti-Flag and NLK in patient-derived GBM cells transduced with Mock control, NLK-WT or NLK Kinase-Negative (K/N) mutant vector. I. Comparison on the effects of NLK-WT and NLK K/N on in vitro proliferation. J. Limiting dilution assays (LDA) for in vitro tumor sphere formation.

Together, these results indicate a negative role of NLK in GBM clonogenic growth and stem cell-like properties.

Previous studies showed that NLK-mediated phosphorylation is critical for its downstream effectors
$[17,27,28]$. To examine whether tumor-suppressive role of NLK is dependent on kinase activity, we overexpressed NLK kinase-inactive mutant (NLK K/N) in GBM cells and determined its effects on short-term proliferation 
and clonogenic growth of GBM cells. Results from the MTT assays and limiting dilution sphere forming assays indicated that NLK K/N is ineffective to elicit NLKmediated responses (Figure $2 \mathrm{H}-2 \mathrm{~J}$ ), indicating that the tumor-suppressive role of NLK in GBM is through its kinase activity-dependent manner.

Although 387, 827, and 131 GBM cells have low levels of endogenous NLK, a subset of GBM cells have relatively high levels of NLK, reflecting GBM heterogeneity (Figure S2A). To determine the role of NLK in this subset of GBM, we performed NLK shRNA-mediated knockdown experiments (Figure S2B and S2C). Knockdown of NLK significantly increased clonogenic growth of 047T GBM cells. Expression levels of Nanog and Sox 2 were higher in NLK knockdown cells compared to the control cells. Taken together, these data suggest that NLK impedes the clonogenic growth of GBM cells and stem cell factor expressions in a gene dosage-dependent manner.

\section{NLK downregulates Wnt/beta-catenin signaling pathway activation}

As we found that NLK overexpression impeded GBM proliferation and clonogenic growth, we further evaluated the effects of NLK on cell cycle kinetics and apoptosis. NLK overexpressing GBM cells also induced significant increase in sub G0/G1 populations and levels of activated caspase 3 , suggesting that NLK-overexpressing cells undergo apoptosis (Figure $3 \mathrm{~A}$ and $3 \mathrm{~B}$ ). To investigate the downstream molecular events of NLK signaling in GBM, we surveyed a few molecular effectors including WNT signaling pathway. Previous studies have shown that WNT pathway is activated in GBM and that Wnt/ beta-catenin signaling in GBM contributes to the maintenance of stem-like properties including inhibition of differentiation and invasive growth pattern [29-33]. As NLK is implicated as a negative regulator of Wnt signaling by interacting with and suppressing transcriptional activity of TCF/LEF family proteins [34], we suspected that NLK negatively regulates Wnt signaling pathway activity in GBM as well. To test this hypothesis, we overexpressed NLK in GBM cells and examined the expression levels of WNT pathway associated genes. Immunoblot analyses showed that expression levels of Wnt pathway associated proteins including LEF-1, CyclinD1, and c-Myc were significantly lower in NLK overexpressing cells compared to the control (Figure 3C). Consistent with these results, WNT responsive promoter luciferase assays showed that NLK overexpression attenuated TCF/LEF-mediated transcriptional activity of GBM cells (Figure 3D). As we observed significant apoptosis in NLK-overexpressing GBM cells, we performed time-course experiments to determine kinetics of apoptosis and WNT inhibition upon NLK overexpression. As TCF/LEF reporter expresses GFP driven by TCF/LEF binding sites, we examined the presence of GFP positive cells with Annexin V-positive apoptotic cells through Fluorescence-activated cell sorting (FACS). Control cells have high WNT activity, as most of the cells were GFP positive. Our results from two different GBM cells showed that NLK overexpression significantly decreased GFP positive cells within a day or two, while proportion of apoptotic cells significantly increased at day 5 of NLK overexpression (Figure 3E). These results suggest that inactivation of WNT pathway is a key molecular event in NLK overexpression. To further characterize the effects of NLK on Wnt pathway activation, we determined mRNA expression levels of known WNT target genes by real time RT-PCR analysis (Figure 3F). Two different GBM cells that overexpress NLK have significantly low levels of the representative WNT associated and target genes including DKK1 and Myc compared to the control cells. Conversely, NLK knockdown significantly upregulated the expression of WNT pathway genes in 047T GBM cells which have a high level of endogenous NLK (Figure S3A).

As NLK suppresses growth properties of GBM through a kinase activity-dependent manner (Figure 2H-2J), we examined the effect of kinaseinactive NLK mutant on Wnt downstream pathway in 131 GBM cells. 131 cells were transduced with the empty vector control, wild type NLK, or NLK mutantexpressing lentivirus, cultured for 2 days, and harvested for immunoblot analysis. Kinase-inactive NLK mutant, in contrast to the wild type NLK, was unable to suppress Wnt pathway associated factors (Figure 3G). In addition, TCF/LEF-mediated transcriptional activity and WNT target gene expression levels were unaffected by NLK $\mathrm{K} / \mathrm{N}$ (Figure $3 \mathrm{G}$ and $3 \mathrm{H}$; Figure $\mathrm{S} 3 \mathrm{~B}$ ). As LEF-1 phosphorylation by NLK is a mechanism to downregulate WNT pathway activation, we determined phosphorylation status of LEF-1 upon ectopic expression of NLK. Elevated level of phosphorylated LEF-1 was found in GBM cells with NLK but not with NLK K/N mutant (Figure 3G). To evaluate the effect of LEF-1 in NLK signaling, we performed functional rescue experiments. As shown above, NLK overexpression significantly decreased proliferation, clonogenic growth and Wnt target gene activities. However, co-expression of NLK and the LEF1-2A mutant, a constitutively active mutant that cannot be phosphorylated by NLK, completely blocked NLKmediated changes, suggesting that LEF-1 is a key mediator for NLK signaling in GBM (Figure 3I-3M). Collectively, these data implicate that NLK negatively regulates canonical Wnt signaling pathway in primary GBM.

\section{NLK negatively regulates mesenchymal activity}

As the recent genomic studies on large sets of GBM indicated distinct GBM subtypes based on their unique genomic alteration and gene expression patterns $[35,36]$, we determined the potential correlations 


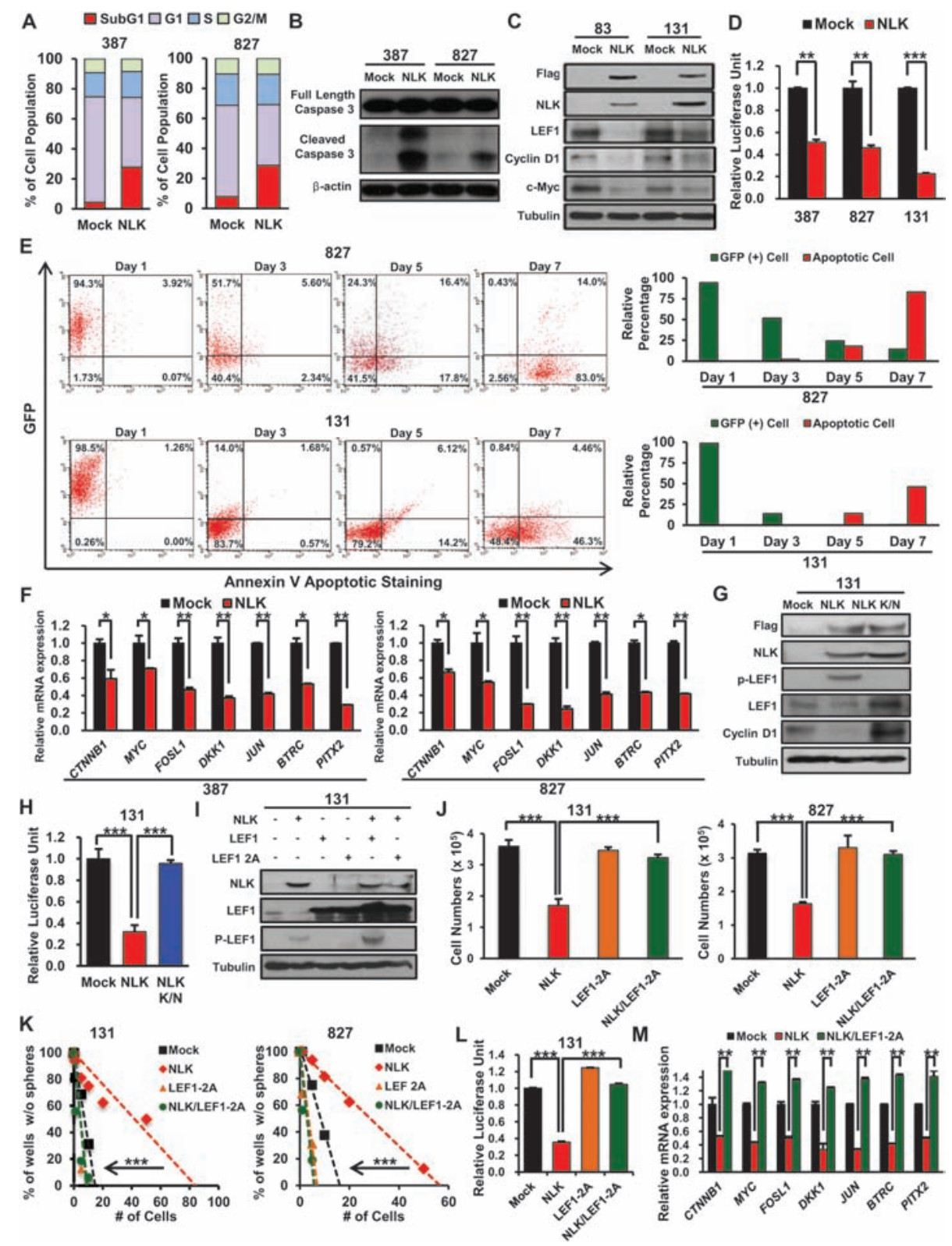

Figure 3: NLK negatively regulates Wnt signaling pathway and its downstream targets. A. Mock and NLK-WT cells have been stained with PI and cell cycle was examined by flow cytometry. $P$-values were calculated using the Fisher's exact test $(p<0.01)$. B. Immunoblots of Caspase 3 in GBM cells transduced with Mock or NLK-WT. C. Immunoblots of Wnt related proteins; anti-Flag, NLK, LEF1, Cyclin D1, and c-Myc. D. The luciferase reporter assay was used to study TCF/LEF promoter activity in Mock or NLK-WT GBM cells. ( $\mathrm{SD}, n=3$ ). E. Flow cytometric analysis of GFP and apoptosis in NLK-WT GBM Cells. GBM cells were cultured and analyzed after 1, 3, 5, or 7 days post NLK-WT transduction. Bar graph represents relative GFP positive and apoptotic cell percentage in the given days. F. Real-time RT-PCR of NLK effects on mRNA expression levels of beta-catenin and its downstream target genes (MYC, FOSll, $D K K 1, J U N, B T R C$, and PITX2). G. Immunoblots of Wnt related proteins in mock, NLK-WT, and NLK K/N GBM Cells; anti-Flag, NLK, phosphor-LEF1, LEF1 and Cyclin D1. H. Relative luciferase activities of mock, NLK-WT, and NLK K/N GBM cells I. Immunoblots of NLK, LEF1 and phosphor-LEF1 in GBM cells transduced with mock, NLK-WT, LEF1, LEF1-2A mutant, NLK-WT/LEF1, or NLK-WT/ LEF1-2A mutant. J. Comparison on the effects of mock, NLK, LEF1-2A mutant and NLK/LEF1-2A mutant on in vitro proliferation. $(+\mathrm{SD}, n=5) \mathrm{K}$. Limiting dilution assays (LDA) for in vitro tumor sphere formation. L. Relative Luciferase activities of Mock, NLK-WT, LEF1-2A, and NLK/LEF1-2A GBM cells. M. Real-time PCR analysis of beta-catenin and its downstream target genes.

between expression levels of $N L K$ and GBM subtypes. When we stratified GBMs into four or five subtypes and determined the average NLK mRNA expression levels in each subtype, NLK expression appeared to be the lowest in mesenchymal subgroup, although proneural subtype also showed low expression levels for NLK mRNA (Figure S4A). On the other hand, more detailed analysis revealed that there are distinct subsets of GBMs that have 
especially low NLK mRNA expression levels. When we determined the subtype of these GBMs $(n=24$ out of $165 \mathrm{GBMs}$ ), $75 \%$ of them belonged to mesenchymal subtype. In contrast, GBM subsets $(n=24)$ with the highest NLK mRNA expression among 165 GBMs, about $50 \%$ of them were identified as classical GBM subtype (Figure 4A and 4B). To further analyze the relationship between NLK expression level and mesenchymal activity, we selected NLK ${ }^{\text {high }}$ and NLK $^{\text {low }}$ GBM specimens based on NLK mRNA expression levels and examined their associations with the gene sets that were used to define the mesenchymal GBM subtypes [37] as an indicator for mesenchymal activity. NLK ${ }^{\text {low }}$ GBMs have high mesenchymal activity whereas $\mathrm{NLK}^{\text {high }}$ GBMs have very low mesenchymal activity, indicating a striking inverse correlation (Figure 4C and 4D). These findings are intriguing because mesenchymal activities in GBMs are often associated with worse prognosis and more aggressive behaviors $[36,38]$. To investigate whether loss of NLK may contribute to its pathology, we determined the expression levels of mesenchymal marker genes such as CASP1, TLR2, and RELB $[8,37]$ in GBMs with or without NLK overexpression. Ectopic expression of NLK significantly decreased expression of mesenchymal marker genes, determined by real-time PCR analysis and immunohistochemical analysis (Figure 4E and 4F). Conversely, NLK knockdown in 047T GBM cells that have high NLK expression induced expression of mesenchymal markers (Figure 4G).

In addition, the mesenchymal marker expression levels were also unaffected or elevated by NLK K/N indicating that the mesenchymal activity may be regulated through kinase-dependent manner (Figure S4B). As our data indicate WNT activation as a consequence of NLK loss, we determined a relationship between NLK and Wnt activities in these GBM specimens. As a surrogate indicator of Wnt activity activation, we used the metagene sets that were previously reported [39]. Using the gene lists used for mesenchymal subtypes, we similarly assigned the level of mesenchymal activity for each GBM as well. Strikingly, NLK ${ }^{\text {low }}$ GBMs have very high levels of both WNT and mesenchymal activities, while NLK ${ }^{\text {high }}$ GBMs have low activities (Figure 4H). Intriguingly, this inverse correlation was especially prominent in a subset of GBMs that possess high WNT activity.

To further investigate molecular association between NLK loss and mesenchymal activity, we examined transcriptional regulation of CD44 and YKL40, widely known markers of mesenchymal GBM subtype [36]. Promoter analysis of both CD44 and YKL40 gene revealed putative TCF/LEF binding sites. We overexpressed NLK or NLK K/N mutants in NLK low GBM cells (131 and 83) and performed chromatin immunoprecipitation-PCR (CHIP-PCR) (Figure 4I). Chromatin immunoprecipitation using anti-LEF-1 antibody using the lysates from NLKlow GBM cells pulled down the promoter DNAs of both
CD44 and YKL40. In contrast, CHIP-PCR results using the lysates from NLK overexpressing cells were negative, suggesting that transcription of CD44 and YKL40 in these cells at least is regulated by NLK. To validate these results, we performed FACS analysis on GBM cells with or without NLK overexpression using anti-CD44 antibody. Both 131 and 83 GBM cells have more than $90 \%$ of CD44 positive cells. Upon NLK overexpression, the number of CD44 positive cells and intensity of CD44 staining were significantly decreased (Figure 4J). Consistent with this, immunoblot analysis and immunofluorescence revealed significantly lower expressions of CD44 in NLK-overexpressing cells compared to the control cells (Figure 4K and 4L). Finally, to determine whether NLK loss regulates mesenchymal activity through LEF-1, we determined the expression levels of CD44 and other mesenchymal markers in GBM cells expressing NLK alone, LEF-1 2A mutant, or both NLK and LEF-1 2A mutant (Figure 4M and 4N). NLK overexpression inhibited the expressions of mesenchymal markers, and this inhibition was completely blocked by co-expression of the constitutively active LEF-1 2A mutant, suggesting NLK negatively regulates mesenchymal activity through LEF1. Together, these results support the notion that NLK is a critical regulator for Wnt and mesenchymal activities in GBM.

\section{NLK suppresses GBM tumor growth in vivo}

The above data suggest that NLK negatively affects the proliferation and clonogenic growth of GBM cells in part via down-regulation of WNT activation and mesenchymal activity. To determine the role of NLK in tumor propagation in vivo, we generated intracranial xenograft tumor models derived from the control and NLK-overexpressing GBM cells. Hematoxylin and eosin (H\&E) staining of mouse brain sections showed a significant difference in tumor volumes between the control and the NLK overexpression group (Figure 5A). Immunohistochemcial analysis using representative mesenchymal markers CD44 and Top2A revealed significantly low levels of these proteins in NLKoverexpressing cells (Figure 5A). Immunofluorescence staining of different GBM sections also showed the same trend (Figure 5B). These results are highly consistent with our in vitro data shown above and suggest that NLK suppresses in vivo tumor growth through negatively regulating Wnt and mesenchymal activities. Consistent with decreased proliferation index in NLKoverexpressing tumors (Figure 5B), the mice injected with NLK-overexpressing cells survived much longer (median survival 73 days) than those with the control cells (median survival 44 days, $p<0.005$ ) (Figure 5C). In order to track NLK-overexpressing cells more closely in vivo, we used dual-color competition assays to evaluate the effect of NLK on GBM tumor propagation. Control and 
A

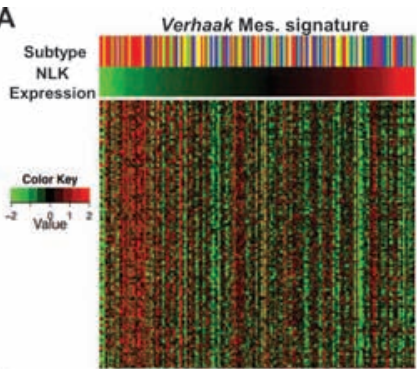

B
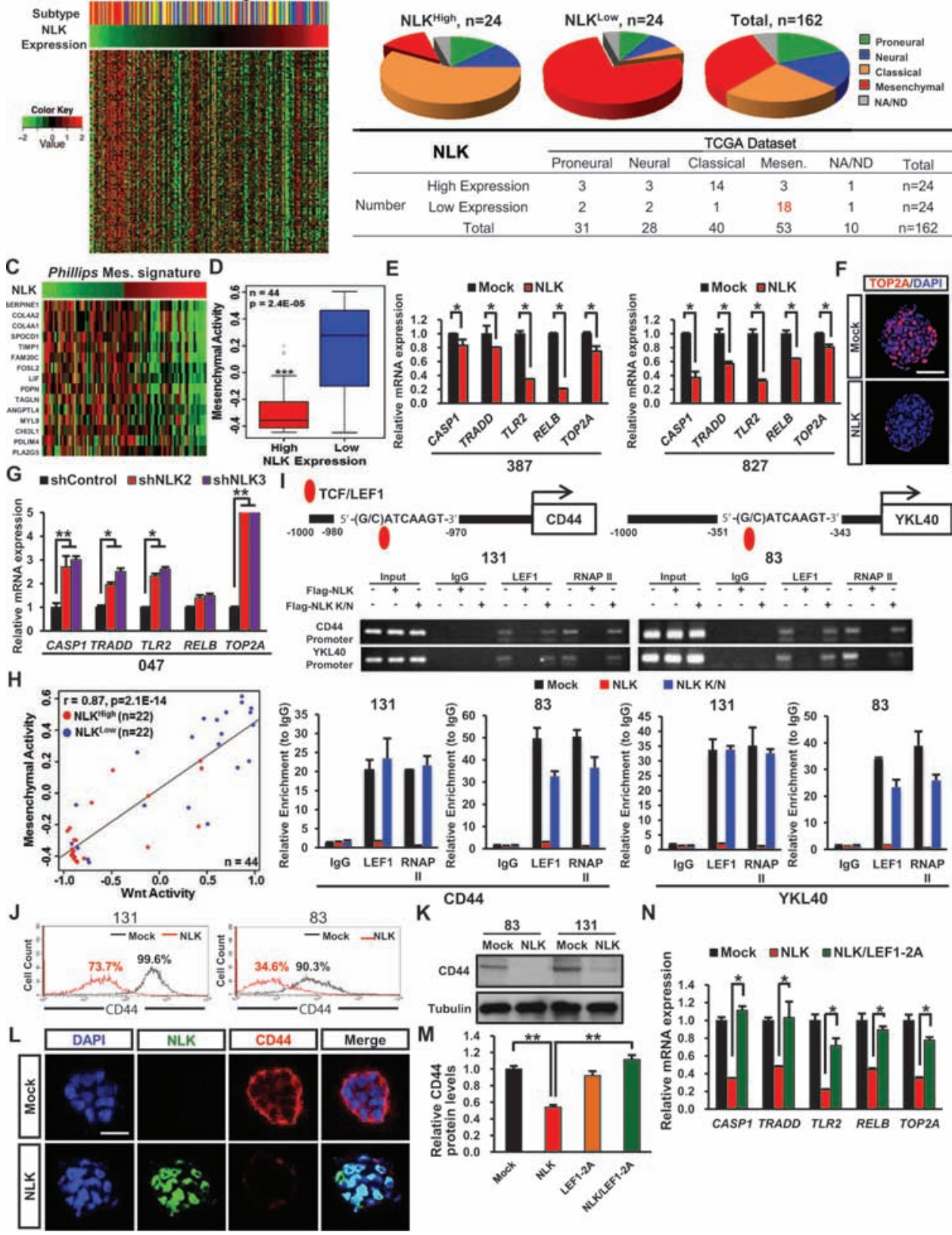

Figure 4: NLK influences on mesenchymal and Wnt activities. A. Heatmap representation of Verhaak mesenchymal-associated gene signature profiles from the TCGA GBM RNA-seq samples according to NLK expression level. $(n=162)$. B. Pie-chart representation of Proneural, Neural, Classical, and Mesenchymal subtypes in TCGA GBM samples in correspondence with NLK ${ }^{\text {high }}$ and NLK ${ }^{\text {low }}$ groups. C. Heatmap representation of Phillips Mesenchyaml-associated gene signature profiles. D. Rembrandt microarray data analysis for Mesenchymal activity in high NLK expressing GBM patients vs. low NLK expressing GBM patients. E. Real-time RT-PCR analysis to determine the mRNA expression levels of Mesenchymal associated genes (CASP1, TRADD, TLR2, RELB, and TOP2A). F. Representative confocal microcopy images of immunofluorescence (IF) staining of TOP2A in spheroids. Scale bar, $100 \mu \mathrm{m}$ G. Real-time RT-PCR analysis of Mesenchymal associated genes. H. Co-expression of Mesenchymal and Wnt activities in high and low NLK expressing groups. $(r=0.87$, $p=2.1 \times 10^{-14}$, Pearson correlation test). I. Chromatin immunoprecipitation (ChIP) analysis of LEF1 on CD44 and YKL40 promoters. J. Flow cytometric analysis of CD44. Gates were drawn based on isotype negative-control staining (not shown). K. Immunoblots of CD44 in mock and NLK-WT GBM Cells. L. Representative confocal images of immunofluorescence (IF) staining of NLK and CD44 in spheroids. Scale bar, $50 \mu \mathrm{m}$. M. Relative CD44 protein levels derived from quantification of immunoblot analysis using ImageJ. N. Realtime RT-PCR analysis of Mesenchymal associated genes.

NLK-overexpressing GBM cells were differentially labeled with GFP- and RFP-expressing lentiviruses, respectively. These tumor cells were mixed to $1: 1$ mixture and co-injected into the brains of mice. More than $95 \%$ of the resultant tumor cells were GFP-positive, indicating that NLK impedes tumor propagation in vivo (Figure 5D). 

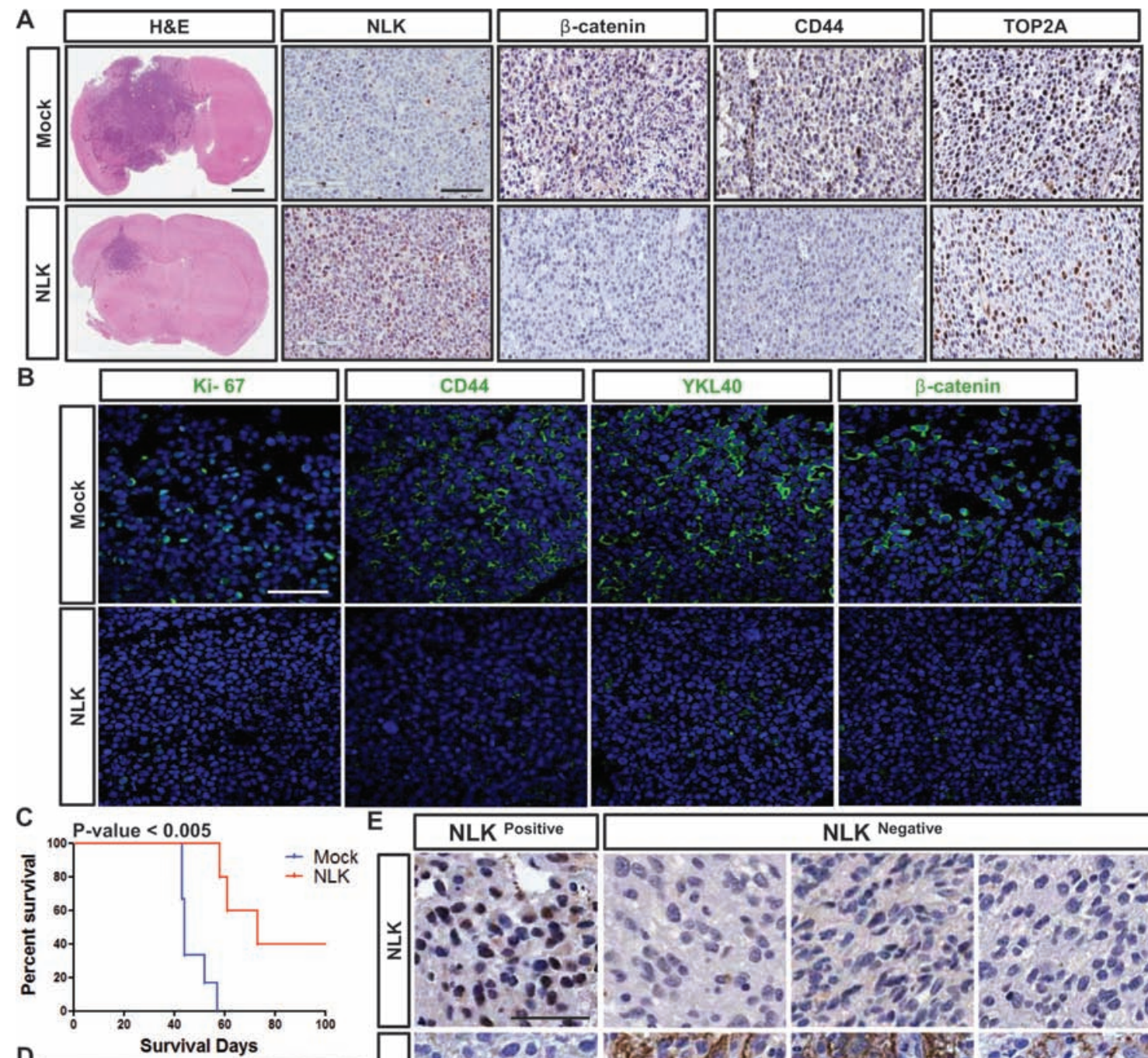

E NLK Positive
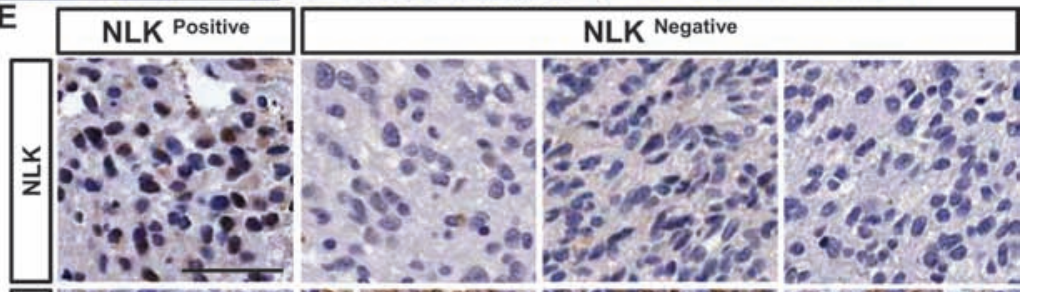

D
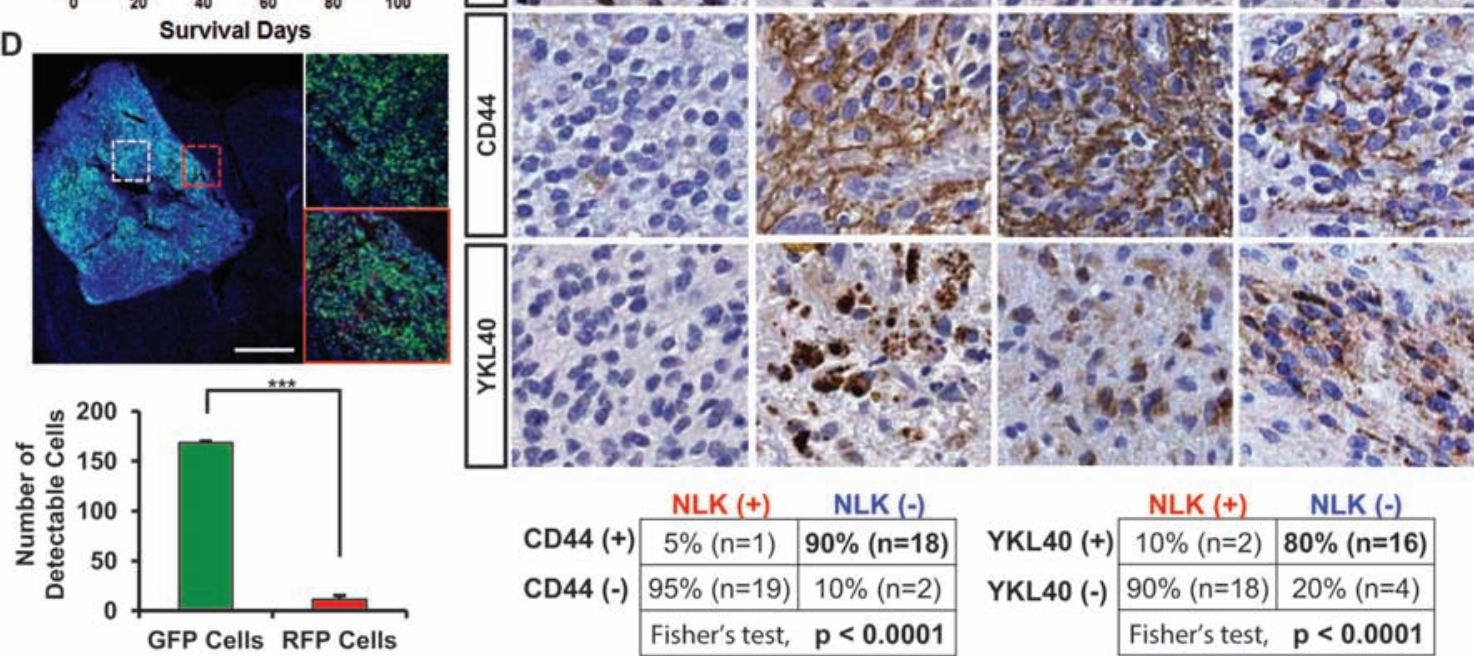

\begin{tabular}{c|c|c|}
\multicolumn{1}{c}{} & \multicolumn{1}{c}{ NLK $(+)$} & \multicolumn{1}{c}{ NLK $(-)$} \\
\cline { 2 - 3 } CD44 (+) & $5 \%(n=1)$ & $90 \%(n=18)$ \\
\cline { 2 - 3 } CD44 (-) & $95 \%(n=19)$ & $10 \%(n=2)$ \\
\cline { 2 - 3 } & Fisher's test, & $p<0.0001$ \\
\cline { 2 - 4 } & &
\end{tabular}

\begin{tabular}{c|c|c|}
\multicolumn{1}{c}{} & \multicolumn{1}{c}{ NLK $(+)$} & \multicolumn{1}{c}{ NLK $(-)$} \\
\cline { 2 - 3 } YKL40 (+) & $10 \%(n=2)$ & $\mathbf{8 0} \%(n=16)$ \\
\cline { 2 - 3 } YKL40 (-) & $90 \%(n=18)$ & $20 \%(n=4)$ \\
\cline { 2 - 3 } & Fisher's test, & $\mathbf{p}<0.0001$ \\
\cline { 2 - 3 } & &
\end{tabular}

Figure 5: NLK overexpression down-regulates mesenchymal signature activity and impedes tumor growth in vivo. A. Representative Immunohistochemical (IHC) images of hematoxylin and eosin (H\&E), NLK, beta-catenin, CD44, and Top2A staining. Scale bars: H\&E, 1mm;rest of the images, $300 \mu \mathrm{m}$. B. Representative Immunofluorescence images of Ki-67, CD44, YKL40, and beta-catenin staining using the frozen sections of tumor bearing brains. Scale bar, $75 \mu \mathrm{m}$. C. Kaplan-Meier survival curves of Mock vs. NLK groups. D. Dual-color competition assay in vivo. A total of 10, 000 cells from a 1:1 mixture of RFP-labeled NLK-WT cells (red) and GFP-labeled Mock cells (green) were implanted into mouse brains. Scale bar, $500 \mu \mathrm{m}$. Bar graph represents the number of GFP and RFP positive cells that were counted in different spots of the tumor that selected randomly. ( $\mathrm{SD}, n=4)$ E. Immunohistochemistry of NLK, CD44 and YKL40 on TMA containing 88 GBM samples and 32 normal brain samples. Scale bar, $100 \mu \mathrm{m}$. 
Finally, to determine the clinical relevance of the NLK loss and mesenchymal activities, we performed TMA IHC analysis using antibodies against mesenchymal markers CD44 and YKL40 (Figure 5E). NLK ${ }^{\text {high }}$ and NLK $^{\text {low }}$ GBM specimens were confirmed by NLK immunohistochemical analysis. Strong inverse correlation between NLK levels and CD44 and/or YKL40 was observed, further supporting that NLK loss is a critical regulator for high mesenchymal activities.

\section{DISCUSSION}

Here we presented a systemic approach for identifying and validating a candidate putative tumor suppressor in GBM, which consists of the candidate gene selection based on genomic copy number alterations and mRNA expression levels of GBM specimen, in vivo loss-of-function screening, and subsequent validation in patient-derived primary GBM models. This approach allowed us to identify NLK as a candidate tumor suppressor and a key regulator of GBM pathogenesis. We showed that restoration of NLK impeded GBM growth in vitro and in vivo, and suppressed WNT signaling, mesenchymal activity, and stem-like features of GBM.

Recent large-scale genomic analyses, especially with high-resolution genomic sequencing and exome sequencing, have identified a large number of novel oncogenes and oncogene-associated mutations, such as IDH1 and histone $3.3[40,41]$. In the case for genes with putative tumor suppressive role, frequencies of inactivating mutations in GBM are generally low except a few well-known bona fide tumor suppressor genes such as p53, NF1, RB, and PTEN. For example, CDKN2A is the well-known tumor suppressor and homozygous deletion of this gene is frequently detected in GBM, but there is little report for inactivating mutation. While promoter methylation and chromatin modification are likely associated with inactivation of putative tumor suppressors, the depth and quantity of the publicly available genomewide data are much smaller than those in genomic alterations and expression profiling studies. Therefore, we utilized the data of genomic copy number alterations and mRNA expression levels of GBM, selected the candidate genes, and screened through in vivo RNAi screen. Tumor suppressors are pivotal modulators of cancer genetics, and it is likely that many of "tumor suppressor" genes may not have inactivating point mutations as a primary cause for functional inactivation. There is a significant lack of functional validation of these candidate genes. The present study represents a step towards this goal. Technical feasibility of in vivo RNAi screen in patientderived primary GBM models shown here suggests that this approach can be also applicable to the search of potential oncogenic effectors in GBM.
NLK is a serine/threonine protein kinase that regulates diverse signaling processes via phosphorylation of several transcription factors [27, 28, 42-44]. We and others have previously shown that aberrant activation of WNT pathway promotes multiple aspects of GBM biology including tumor initiation, proliferation, inhibition of apoptosis, and stem-like features [32, 33, 45, 46]. Despite the prominent roles of WNT pathway in GBM, GBM rarely have mutations in the negative regulators such as APC and NLK. Notably, a subset of GBMs with the lowest NLK expression harbors very high levels of both WNT and mesenchymal activities. It is possible that NLK loss is a causative event in aberrant WNT activation at least in this subset of GBMs. Further studies to investigate GBM evolution process are warranted.

Our data indicate NLK loss in GBM is not completely restricted to mesenchymal subtype. Indeed, we found weaker correlations between NLK loss and the signaling pathways such as TGF-beta, NF- $\kappa$ B, and STAT3, compared to WNT pathway. Mesenchymal features can be driven by multiple factors including tumor genetics and microenvironment. The proneural and the mesenchymal subtypes have been defined as the most consistent subgroups in GBM by transcriptome analysis [6, 36, 37]. However, more recent studies have reported proneural to mesenchymal switching and co-existence of multiple subtypes within a GBM, suggesting a dynamic nature of GBM subtype $[35,36,47,48]$. In this context, causative roles of NLK loss in mesenchymal properties of GBM can be more prominent in a subset of GBMs.

In conclusion, our studies demonstrate in vivo RNAi screening tool as a powerful tool to identify and validate a candidate tumor suppressor. Currently, most WNT pathway targeted drugs have been developed against the ligands, receptors, and an intermediate signaling molecule GSK $3 \beta$. Given the profound effects of NLK overexpression in inhibition of WNT, mesenchymal, and stem cell associated proteins, it is conceivable to develop therapeutic approaches that mimic the function of NLK. Furthermore, NLK level can be a biomarker to predict WNT and/or mesenchymal activities in GBM for the selection of patient groups that are most likely to be benefit from WNT pathway targeting therapy.

\section{MATERIALS AND METHODS}

\section{RNAi screen}

For shRNA screen procedure, different sets of cells were infected with a pool of approximately 200 lentiviral shRNAs targeting 24 human genes at a representation of $\sim 500$ cells per shRNAs at Multiplicity of Infection at 1 . On day 2 of the post infection, puromycin (Sigma) $(1 \mathrm{ug} / \mathrm{ml})$ was added to remove any non-infected cells and the selection procedure proceeded for the next 3 days. 
Afterwards, 100,000 transduced cells were injected into its recipient mice and the Control populations were harvested. For each corresponding samples, shRNA barcodes were PCR-recovered from genomic samples, and analyzed through deep sequencing technology (Illumina High-Seq 2000). Each shRNA read was normalized to its whole population and changes in the relative abundance of each shRNA in the library were measured.

\section{Patient-derived GBM specimens and primary cell culture}

Following informed consent in accordance with the appropriate Institutional Review Boards, glioblastoma specimens were obtained from patients undergoing surgery. Patient-derived GBM cells were cultured in the "NBE" neurosphere culture condition [21]

\section{Orthotopic GBM xenograft models}

Six-week-old female BALB/c nude mice (Orient Bio, Seoul, South Korea) were used for intracranial injection. Patient-derived GBM cells were injected into the brains of mice by stereotactic intracranial injection (coordinates: $2 \mathrm{~mm}$ anterior, $2 \mathrm{~mm}$ lateral, $2.5 \mathrm{~mm}$ depth from the dura). Mice were killed either when $25 \%$ body weight loss or neurological symptoms (lethargy, ataxia, and seizures) were observed. All mice experiments were performed according to the Association for Assessment and Accreditation of Laboratory Animal Care-accredited guidelines of our institute's Animal Use and Care Committee.

\section{TMA and tumor samples}

For analysis of NLK levels by immunohistochemistry, TMA containing glioblastoma and normal brain tissues were used. The TMA slides contain 88 cases of glioblastoma and 31 of their own accordant normal brain tissue parts. Brain tissue samples were fixed by formalin and embedded in paraffin; then sections of paraffin-embedded glioma specimens were stained with an antibody against human NLK (Sigma-Aldrich), CD44 (Sigma-Aldrich), and YKL40 (Life-Science).

\section{Lentivirus production and transduction}

To generate recombinant lentivirus, a cloning package including entry (pCR8/GW/TOPO) and lentiviral destination ( $\mathrm{pLenti6/V5-Dest)} \mathrm{vectors} \mathrm{were}$ used (Invitrogen). 293FT cells were transfected using lipofectamine 2000 (Invitrogen) for the production of lentiviruses. After the initial transfection, supernatant was collected after 24, 48, 72 hours respectively and were concentrated. For the transduction of GBM cells, lentiviruses were added into the culture medium for 2 days and then blasticidin $(3 \sim 4 \mathrm{ug} / \mathrm{ml})$ selection was performed to eliminate any non-infected cells.

\section{Quantitative real-time RT-PCR}

RNAs were extracted (Qiagen) and their complementary DNAs were synthesized (Invitrogen) per manufacturers" instructions. Real-time RT-PCR was performed using primers listed in Supplementary Table 2 (7500 Fast Real-Time PCR System). Duplicate reactions were performed for each set of primers and the relative amounts of target transcripts were normalized to the number of human beta-actin transcripts. The relative quantification of target gene expression was performed with the comparative cycle threshold (CT) method.

\section{Cell proliferation assay and neurosphere forminglimiting dilution assay}

Cell proliferation was measured using EZ-cytox cell viability kit (DAEIL Lab, Seoul, South Korea) according to the manufacturer's protocol. A total of $1 \times 10^{4}$ cells were plated per well in a 96-well plate, and each sample were plated in quintuplicate. After 7 days, Ez-cytox was added into each well accordant to its appropriate volume and incubated for three hours. At the end of the incubation, cell viability was evaluated by measuring the optical density at $450 \mathrm{~nm}$. For neurosphere forming limiting diluation assay, cells were seeded at a range of 1-200 cells per well. After 1 2 weeks, the number of wells without spheres were counted. LDA clonogenic index was calculated as the inverse of the $\mathrm{x}$-intercept of the regression between the number of wells without spheres and the number of cells seeded.

\section{Immunofluorescence analysis}

Cells were fixed in 4\% paraformaldehyde in PBS for 20 minutes and blocked with 5\% donkey serum. Afterwards, they were incubated with primary antibodies over night at $4^{\circ} \mathrm{C}$ and then with anti-mouse Alexa 594 (Invitrogen) and anti-rabbit Alexa 647 (Invitrogen) secondary antibodies for 2 hrs with washing in between. Microscopy was done with a confocal microscope.

\section{Western blot assay}

Cells were lysed in RIPA buffer supplemented with proteinase inhibitor and phosphatase inhibitor. Total proteins $(15 \sim 30 \mu \mathrm{g} /$ lane $)$ were separated by SDS-PAGE and transferred to PVDF membranes (Millipore). The blots were blocked for $1 \mathrm{hr}$ in 5\% Bovine Serum Albumin in TBS-T and were incubated with mouse anti-V5 NLK (Invitrogen), NLK (Sigma-Aldrich) Sox2 (R\&D Systems), Nanog (Cell Signaling), LEF1 (Cell Signaling), C-myc(Cell Signaling), p-LEF1 (Millipore), anti-FLAG 
(Cell Signaling), and Cyclin D1 (Cell Signaling) overnight at $4{ }^{\circ} \mathrm{C}$. After washing with TBS-T, the blots were incubated with HRP-conjugated secondary antibody for $1 \mathrm{hr}$ at room temperature. Detection was performed using the chemiluminescence method (ECL, GE Healthcare).

\section{Luciferase assay}

GBM cells from a reporter tumor were transduced with appropriate lentivirus. After 2 days, blasticidin was added to eliminate any uninfected cells. Post 3 days of blasticidin (Invitrogen) selection, total cell extracts were prepared using GloLysis Buffer, and the luciferase activities were analyzed using the Steady-Glo Luciferase Assay System accordance with the manufacturer's protocol (Promega, USA). Each value was normalized to its total protein amounts and TCF/LEF luciferase value was normalized to mCMV backbone luciferase value.

\section{Flow cytometry}

Cells were fixed and permeabilized using solutions from BD Biosciences. They were stained with different primary antibodies with their accordance purposes; CD44 (Sigma-Aldrich), and Annexin V (eBioscience). Afterwards, they were stained with Alexa 647 (Invitrogen) secondary antibodies. Flow cytometry was performed in FACS Calibur (BD Biosciences).

\section{Chromatin immunoprecipitation (ChIP) assay}

Chromatin immunoprecipitation (ChIP) was carried out using EZ ChIP kit (Millipore) following the manufacturer's protocol. Briefly, the cells were formaldehyde crosslinked and the lysates were shared by ultrasonication and cleared by centrifugation and diluted in ChIP dilution buffer. IP complexes were immunoprecipitated with LEF1 antibody (Santa Cruz), normal Goat IgG (negative control) and RNA pol II (positive control). Crosslinks were reserved by incubating chromatin at $65^{\circ} \mathrm{C}$ overnight and enriched DNA was amplified by PCR and primers indicated below.

$\begin{array}{ccc}\text { CD44 } & \text { promoter } & \text { forward, } \\ \text { CTGCGTTTGATTTCCAAACA }-3^{\prime} \text { and } & \\ \text { CD44 } & \text { promoter } & \text { reverse, } \\ \text { CCTACCCAGCAGATCTTAAAGAGAGG }-3^{\prime}, \\ \text { YKL40 promoter } & \text { forward, } \\ \text { CTGTTCACCCCTCCCCTAACACT }-3^{\prime} & \\ \text { YKL40 } & \text { promoter } & \text { reverse, } \\ \text { GGCTGAAAATCTGTCTATTCTTCTG }-3^{\prime} .\end{array}$

\section{Statistical analysis}

All statistical analysis was conducted using Student's $t$ test to determine the significance of results. Overall survival curves were plotted according to the
Kaplan-Meier method. All differences were considered to be statistically significant at the level of $P<0.05$, $* P<0.01, * * P<0.001 ; * * *$

\section{ACKNOWLEDGMENTS}

This research was supported by the grant of Korea Health Technology R\&D Project through the Korea Health Industry Development Institute (KHIDI), funded by the Ministry of Health \& Welfare, Republic of Korea (HI14C3418). Additional supports were from the Global Frontier Project grant (NRF-2012M3A6A-2010-0029781) of National Research Foundation funded by the Ministry of Science, NIH R01 NS082312 and R01 NS 083767 (JL), ICT and Future Planning (MSIP) of Korea and Samsung Medical Center grant (GFO1140011).

\section{Editorial note}

This paper has been accepted based in part on peerreview conducted by another journal and the authors' response and revisions as well as expedited peer-review in Oncotarget.

\section{REFERENCES}

1. Louis DN, Ohgaki H, Wiestler OD, Cavenee WK, Burger PC, Jouvet A, Scheithauer BW, Kleihues P. The 2007 WHO classification of tumours of the central nervous system. Acta neuropathologica. 2007; 114:97-109.

2. Furnari FB, Fenton T, Bachoo RM, Mukasa A, Stommel JM, Stegh A, Hahn WC, Ligon KL, Louis DN, Brennan C, Chin L, DePinho RA, Cavenee WK. Malignant astrocytic glioma: genetics, biology, and paths to treatment. Genes \& development. 2007; 21:2683-2710.

3. Stupp R, Mason WP, van den Bent MJ, Weller M, Fisher B, Taphoorn MJ, Belanger K, Brandes AA, Marosi C, Bogdahn U, Curschmann J, Janzer RC, Ludwin SK, Gorlia T, Allgeier A, Lacombe D, et al. Radiotherapy plus concomitant and adjuvant temozolomide for glioblastoma. The New England journal of medicine. 2005; 352:987-996.

4. Vassilev LT, Vu BT, Graves B, Carvajal D, Podlaski F, Filipovic Z, Kong N, Kammlott U, Lukacs C, Klein C, Fotouhi N, Liu EA. In vivo activation of the p53 pathway by small-molecule antagonists of MDM2. Science. 2004; 303:844-848.

5. Cancer Genome Atlas Research N . Comprehensive genomic characterization defines human glioblastoma genes and core pathways. Nature. 2008; 455:1061-1068.

6. Brennan CW, Verhaak RG, McKenna A, Campos B, Noushmehr H, Salama SR, Zheng S, Chakravarty D, Sanborn JZ, Berman SH, Beroukhim R, Bernard B, Wu CJ, Genovese G, Shmulevich I, Barnholtz-Sloan J, et al. 
The somatic genomic landscape of glioblastoma. Cell. 2013; 155:462-477.

7. Hata N, Yoshimoto K, Yokoyama N, Mizoguchi M, Shono T, Guan Y, Tahira T, Kukita Y, Higasa K, Nagata S, Iwaki T, Sasaki T, Hayashi K. Allelic losses of chromosome 10 in glioma tissues detected by quantitative singlestrand conformation polymorphism analysis. Clinical chemistry. 2006; 52:370-378.

8. Joo KM, Kim J, Jin J, Kim M, Seol HJ, Muradov J, Yang H, Choi YL, Park WY, Kong DS, Lee JI, Ko YH, Woo HG, Lee J, Kim S, Nam DH. Patient-specific orthotopic glioblastoma xenograft models recapitulate the histopathology and biology of human glioblastomas in situ. Cell reports. 2013; 3:260-273.

9. Zender L, Xue W, Zuber J, Semighini CP, Krasnitz A, Ma B, Zender P, Kubicka S, Luk JM, Schirmacher P, McCombie WR, Wigler M, Hicks J, Hannon GJ, Powers S, Lowe SW. An oncogenomics-based in vivo RNAi screen identifies tumor suppressors in liver cancer. Cell. 2008; 135:852-864.

10. Yamada KM, Araki M. Tumor suppressor PTEN: modulator of cell signaling, growth, migration and apoptosis. Journal of cell science. 2001; 114:2375-2382.

11. Wechsler DS, Shelly CA, Petroff CA, Dang CV. MXI1, a putative tumor suppressor gene, suppresses growth of human glioblastoma cells. Cancer research. 1997; 57:4905-4912.

12. Garcia-Cao I, Song MS, Hobbs RM, Laurent G, Giorgi C, de Boer VC, Anastasiou D, Ito K, Sasaki AT, Rameh L, Carracedo A, Vander Heiden MG, Cantley LC, Pinton P, Haigis MC, Pandolfi PP. Systemic elevation of PTEN induces a tumor-suppressive metabolic state. Cell. 2012; 149:49-62.

13. Manni I, Tunici P, Cirenei N, Albarosa R, Colombo BM, Roz L, Sacchi A, Piaggio G, Finocchiaro G. Mxi1 inhibits the proliferation of U87 glioma cells through downregulation of cyclin B1 gene expression. British journal of cancer. 2002; 86:477-484.

14. Huang Y, Jiang Y, Lu W, Zhang Y. Nemo-like kinase associated with proliferation and apoptosis by c-Myb degradation in breast cancer. PloS one. 2013; 8:e69148.

15. Yasuda J, Tsuchiya A, Yamada T, Sakamoto M, Sekiya T, Hirohashi S. Nemo-like kinase induces apoptosis in DLD-1 human colon cancer cells. Biochemical and biophysical research communications. 2003; 308:227-233.

16. Lv L, Wan C, Chen B, Li M, Liu Y, Ni T, Yang Y, Liu Y, Cong X, Mao G, Xue Q. Nemo-like kinase (NLK) inhibits the progression of NSCLC via negatively modulating WNT signaling pathway. Journal of cellular biochemistry. 2014; 115:81-92.

17. Ishitani $T$, Ninomiya-Tsuji $J$, Nagai $S$, Nishita $M$, Meneghini M, Barker N, Waterman M, Bowerman B, Clevers H, Shibuya H, Matsumoto K. The TAK1NLK-MAPK-related pathway antagonizes signalling between beta-catenin and transcription factor TCF. Nature. 1999; 399:798-802.

18. Rhodes DR, Yu J, Shanker K, Deshpande N, Varambally R, Ghosh D, Barrette T, Pandey A, Chinnaiyan AM. ONCOMINE: a cancer microarray database and integrated data-mining platform. Neoplasia. 2004; 6:1-6.

19. Madhavan S, Zenklusen JC, Kotliarov Y, Sahni H, Fine HA, Buetow K. Rembrandt: helping personalized medicine become a reality through integrative translational research. Molecular cancer research : MCR. 2009; 7:157-167.

20. Dirks PB. Brain tumor stem cells: the cancer stem cell hypothesis writ large. Molecular oncology. 2010; 4:420-430.

21. Lee J, Kotliarova S, Kotliarov Y, Li A, Su Q, Donin NM, Pastorino S, Purow BW, Christopher N, Zhang W, Park JK, Fine HA. Tumor stem cells derived from glioblastomas cultured in bFGF and EGF more closely mirror the phenotype and genotype of primary tumors than do serum-cultured cell lines. Cancer cell. 2006; 9:391-403.

22. Singh SK, Hawkins C, Clarke ID, Squire JA, Bayani J, Hide T, Henkelman RM, Cusimano MD, Dirks PB. Identification of human brain tumour initiating cells. Nature. 2004; 432:396-401.

23. Hemmati HD, Nakano I, Lazareff JA, Masterman-Smith M, Geschwind DH, Bronner-Fraser M, Kornblum HI. Cancerous stem cells can arise from pediatric brain tumors. Proceedings of the National Academy of Sciences of the United States of America. 2003; 100:15178-15183.

24. Mangiola A, Lama G, Giannitelli C, De Bonis P, Anile C, Lauriola L, La Torre G, Sabatino G, Maira G, JhanwarUniyal M, Sica G. Stem cell marker nestin and c-Jun $\mathrm{NH} 2$-terminal kinases in tumor and peritumor areas of glioblastoma multiforme: possible prognostic implications. Clinical cancer research: an official journal of the American Association for Cancer Research. 2007; 13:6970-6977.

25. Gangemi RM, Griffero F, Marubbi D, Perera M, Capra MC, Malatesta P, Ravetti GL, Zona GL, Daga A, Corte G. SOX2 silencing in glioblastoma tumor-initiating cells causes stop of proliferation and loss of tumorigenicity. Stem cells. 2009; 27:40-48.

26. Stiles CD, Rowitch DH. Glioma stem cells: a midterm exam. Neuron. 2008; 58:832-846.

27. Ohkawara B, Shirakabe K, Hyodo-Miura J, Matsuo R, Ueno N, Matsumoto K, Shibuya H. Role of the TAK1NLK-STAT3 pathway in TGF-beta-mediated mesoderm induction. Genes \& development. 2004; 18:381-386.

28. Kanei-Ishii C, Ninomiya-Tsuji J, Tanikawa J, Nomura T, Ishitani T, Kishida S, Kokura K, Kurahashi T, Ichikawa-Iwata E, Kim Y, Matsumoto K, Ishii S. Wnt-1 signal induces phosphorylation and degradation of c-Myb protein via TAK1, HIPK2, and NLK. Genes \& development. 2004; 18:816-829.

29. Zheng H, Ying H, Wiedemeyer R, Yan H, Quayle SN, Ivanova EV, Paik JH, Zhang H, Xiao Y, Perry SR, Hu J, 
Vinjamoori A, Gan B, Sahin E, Chheda MG, Brennan C, et al. PLAGL2 regulates Wnt signaling to impede differentiation in neural stem cells and gliomas. Cancer cell. 2010; 17:497-509.

30. Jin X, Jeon HY, Joo KM, Kim JK, Jin J, Kim SH, Kang BG, Beck S, Lee SJ, Kim JK, Park AK, Park WY, Choi YJ, Nam DH, Kim H. Frizzled 4 regulates stemness and invasiveness of migrating glioma cells established by serial intracranial transplantation. Cancer research. 2011; 71:3066-3075.

31. Zhang N, Wei P, Gong A, Chiu WT, Lee HT, Colman H, Huang H, Xue J, Liu M, Wang Y, Sawaya R, Xie K, Yung WK, Medema RH, He X, Huang S. FoxM1 promotes beta-catenin nuclear localization and controls Wnt targetgene expression and glioma tumorigenesis. Cancer cell. 2011; 20:427-442.

32. Kim KH, Seol HJ, Kim EH, Rheey J, Jin HJ, Lee Y, Joo KM, Lee J, Nam DH. Wnt/beta-catenin signaling is a key downstream mediator of MET signaling in glioblastoma stem cells. Neuro-oncology. 2013; 15:161-171.

33. Kim Y, Kim KH, Lee J, Lee YA, Kim M, Lee SJ, Park K, Yang H, Jin J, Joo KM, Lee J, Nam DH. Wnt activation is implicated in glioblastoma radioresistance. Laboratory investigation; a journal of technical methods and pathology. 2012; 92:466-473.

34. Emami KH, Brown LG, Pitts TE, Sun X, Vessella RL, Corey E. Nemo-like kinase induces apoptosis and inhibits androgen receptor signaling in prostate cancer cells. The Prostate. 2009; 69:1481-1492.

35. Bhat KP, Balasubramaniyan V, Vaillant B, Ezhilarasan R, Hummelink K, Hollingsworth F, Wani K, Heathcock L, James JD, Goodman LD, Conroy S, Long L, Lelic N, Wang S, Gumin J, Raj D, et al. Mesenchymal differentiation mediated by NF-kappaB promotes radiation resistance in glioblastoma. Cancer cell. 2013; 24:331-346.

36. Phillips HS, Kharbanda S, Chen R, Forrest WF, Soriano RH, Wu TD, Misra A, Nigro JM, Colman H, Soroceanu L, Williams PM, Modrusan Z, Feuerstein BG, Aldape K. Molecular subclasses of high-grade glioma predict prognosis, delineate a pattern of disease progression, and resemble stages in neurogenesis. Cancer Cell. 2006; 9:157-173.

37. Verhaak RG, Hoadley KA, Purdom E, Wang V, Qi Y, Wilkerson MD, Miller CR, Ding L, Golub T, Mesirov JP, Alexe G, Lawrence M, O'Kelly M, Tamayo P, Weir BA, Gabriel S, et al. Integrated genomic analysis identifies clinically relevant subtypes of glioblastoma characterized by abnormalities in PDGFRA, IDH1, EGFR, and NF1. Cancer cell. 2010; 17:98-110.

38. Day BW, Stringer BW, Al-Ejeh F, Ting MJ, Wilson J, Ensbey KS, Jamieson PR, Bruce ZC, Lim YC,
Offenhauser C, Charmsaz S, Cooper LT, Ellacott JK, Harding A, Leveque L, Inglis P, et al. EphA3 maintains tumorigenicity and is a therapeutic target in glioblastoma multiforme. Cancer cell. 2013; 23:238-248.

39. Nguyen DX, Chiang AC, Zhang XH, Kim JY, Kris MG, Ladanyi M, Gerald WL, Massague J. WNT/TCF signaling through LEF1 and HOXB9 mediates lung adenocarcinoma metastasis. Cell. 2009; 138:51-62.

40. Parsons DW, Jones S, Zhang X, Lin JC, Leary RJ, Angenendt P, Mankoo P, Carter H, Siu IM, Gallia GL, Olivi A, McLendon R, Rasheed BA, Keir S, Nikolskaya T, Nikolsky Y, et al. An integrated genomic analysis of human glioblastoma multiforme. Science. 2008; 321:1807-1812.

41. Sturm D, Witt H, Hovestadt V, Khuong-Quang DA, Jones DT, Konermann C, Pfaff E, Tonjes M, Sill M, Bender S, Kool M, Zapatka M, Becker N, Zucknick M, Hielscher T, Liu XY, et al. Hotspot mutations in H3F3A and IDH1 define distinct epigenetic and biological subgroups of glioblastoma. Cancer cell. 2012; 22:425-437.

42. Cadigan KM, Nusse R. Wnt signaling: a common theme in animal development. Genes \& development. 1997; 11:3286-3305.

43. Ishitani $\mathrm{T}$, Hirao $\mathrm{T}$, Suzuki $\mathrm{M}$, Isoda $\mathrm{M}$, Ishitani $\mathrm{S}$, Harigaya K, Kitagawa M, Matsumoto K, Itoh M. Nemo-like kinase suppresses Notch signalling by interfering with formation of the Notch active transcriptional complex. Nature cell biology. 2010; 12:278-285.

44. Ishitani T, Ishitani S. Nemo-like kinase, a multifaceted cell signaling regulator. Cellular signalling. 2013; 25:190-197.

45. Palos TP, Zheng S, Howard BD. Wnt signaling induces GLT-1 expression in rat C6 glioma cells. Journal of neurochemistry. 1999; 73:1012-1023.

46. Satoh J, Kuroda Y. Beta-catenin expression in human neural cell lines following exposure to cytokines and growth factors. Neuropathology: official journal of the Japanese Society of Neuropathology. 2000; 20:113-123.

47. Ozawa T, Riester M, Cheng YK, Huse JT, Squatrito M, Helmy K, Charles N, Michor F, Holland EC. Most human non-GCIMP glioblastoma subtypes evolve from a common proneural-like precursor glioma. Cancer cell. 2014; 26:288-300.

48. Patel AP, Tirosh I, Trombetta JJ, Shalek AK, Gillespie SM, Wakimoto H, Cahill DP, Nahed BV, Curry WT, Martuza RL, Louis DN, Rozenblatt-Rosen O, Suva ML, Regev A, Bernstein BE. Single-cell RNA-seq highlights intratumoral heterogeneity in primary glioblastoma. Science. 2014; 344:1396-1401. 